\title{
In vitro assessment of drug delivery through an endotracheal tube using a dry powder inhaler delivery system
}

\author{
Mark L Everard, Sunalene G Devadason, Peter N Le Souëf
}

\begin{abstract}
Background - Jet nubulisers and metered dose inhalers are widely used to deliver aerosolised drugs to the lungs of intubated patients in adult intensive care units. Drug delivery using these systems has been shown to be inefficient and both forms of delivery have the potential to induce paradoxical bronchoconstriction in patients with reactive airways disease.

Methods - Experiments were carried out to determine whether it was possible to deliver drug from a dry powder delivery system through an endotracheal tube. A $200 \mu \mathrm{g}$ budesonide Turbohaler was enclosed in a chamber which allowed it to be inserted into a ventilator circuit. Experiments were performed with a multistage liquid impinger in which drug was drawn through the Turbohaler and endotracheal tube at $60 \mathrm{1} / \mathrm{min}$ providing an index of the maximum drug delivery achievable via this route. A second series of experiments was performed in which the Turbohaler was placed in a ventilator circuit using a Servo $900 \mathrm{C}$ volume cycled ventilator. Drug delivered from the Turbohaler during the inspiratory phase was collected on a filter placed between the end of a $9 \mathrm{~mm}$ endotracheal tube and a model lung. A tidal volume of $500 \mathrm{ml}$ and inspiratory time of 0.5 seconds was used. Budesonide was assayed using an ultraviolet spectrophotometric assay.
\end{abstract}

Results - Thirty percent of the nominal dose passed through the endotracheal tube and was collected in the multistage liquid impinger. Mean drug delivery to the filter in the ventilator circuit was $20 \%$.

Conclusions - This in vitro study indicates that drugs from dry powder inhalers (in this case the Turbohaler) can be satisfactorily delivered through endotracheal tubes and that clinical evaluation of this technique is now indicated.

(Thorax 1996;51:75-77)

Keywords: aerosol, endotracheal tube, dry powder inhaler.

Jet nebulisers have traditionally been used in adult intensive care units when administering drugs via endotracheal tubes to the lower airways of intubated patients. ${ }^{1-4}$ More recently, many centres - particularly in North America - have been using metered dose inhalers for this purpose. ${ }^{35-9}$ It has been known for some time that the effective drug delivery from conventional jet nebulisers is generally low due to factors such as raining out of drug in the ventilator, ${ }^{1-4}$ while other problems such as cooling of the inspired air, adding gas volume to the ventilator circuit, and poor reproducibility of output have been cited as reasons for considering alternative systems. ${ }^{5710}$ Both dosimetric jet nebulisers, which deliver drug only during inspiration, ${ }^{11}$ and ultrasonic nebulisers ${ }^{12}$ have been shown in in vitro studies to be capable of delivering significantly greater doses, but they have not been widely accepted and no deposition studies have been performed to confirm their promise. Comparisons of metered dose inhaler and jet nebuliser based systems in the intensive care setting have produced conflicting results, with some indicating improved drug delivery using metered dose inhaler systems ${ }^{7}$ and some improved delivery with jet nebulisers. ${ }^{13}$ Reviewing the evidence, it would appear that both metered dose inhaler and jet nebuliser systems, if suitably chosen and used optimally, can deliver therapeutic doses to the lungs of intubated patients, and that the main impetus in North America to move towards the metered dose inhaler based systems is the financial saving, principally in respiratory technologist time, that such systems provide. ${ }^{568}$

One problem common to aerosols generated by both wet nebuliser and metered dose inhaler systems is the ability of the aerosol to induce bronchoconstriction in those with obstructive airways disease. ${ }^{1415}$ The potential for inducing such events in some severely ill asthmatic patients in the intensive care setting is of some concern.

A series of in vitro experiments was performed to determine whether it is possible to deliver budesonide, in the form of a dry powder, via endotracheal tubes using a Turbohaler and a novel adapter for use in ventilator circuits.

\section{Methods}

Budesonide Turbohalers, $200 \mu \mathrm{g}$, were used for these experiments. The standard Turbohaler mouthpiece was modified by cutting back the outer covering to reveal the inner cylinder containing the spiral disaggregation channels, which could then be inserted into the end of an endotracheal tube. The Turbohaler was enclosed in a chamber or "spaceship" (Astra Draco, Lund, Sweden) which permits air to flow through the Turbohaler and out via the 


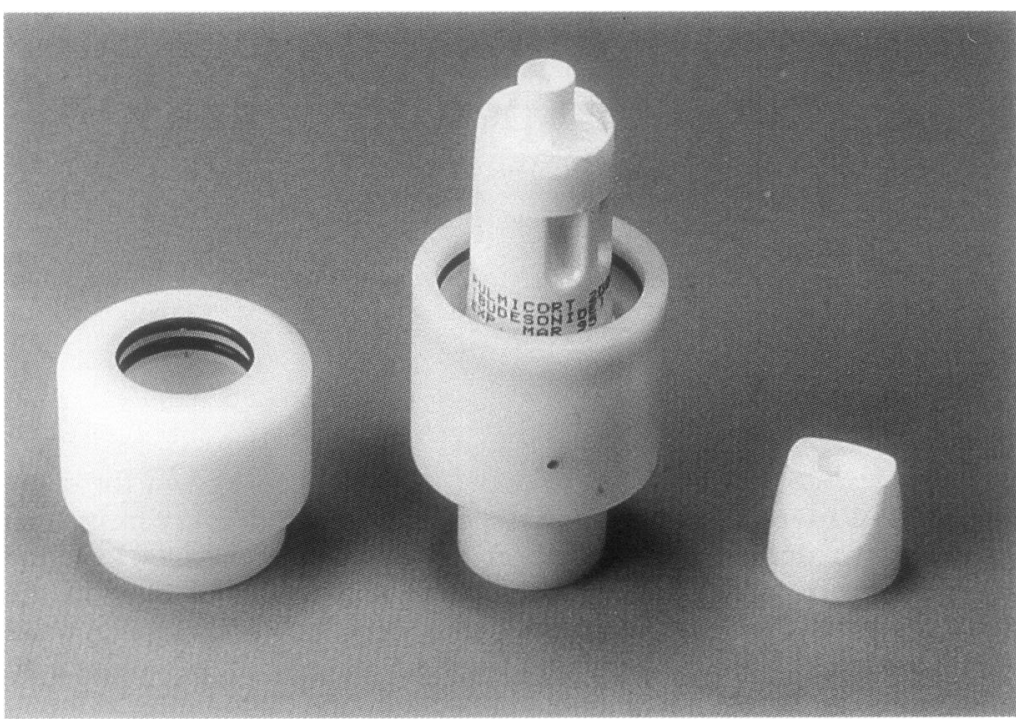

Figure 1 Turbohaler with modified mouthpiece and "spaceship".

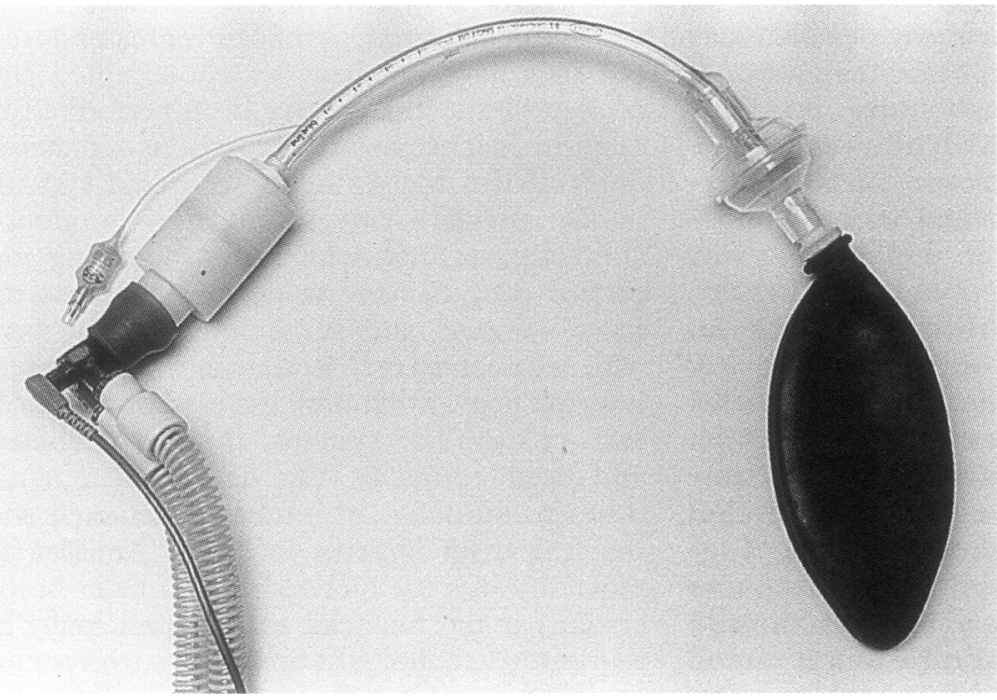

Figure 2 Positioning of the Turbohaler within a ventricular circuit between the manifold and the endotracheal tube.

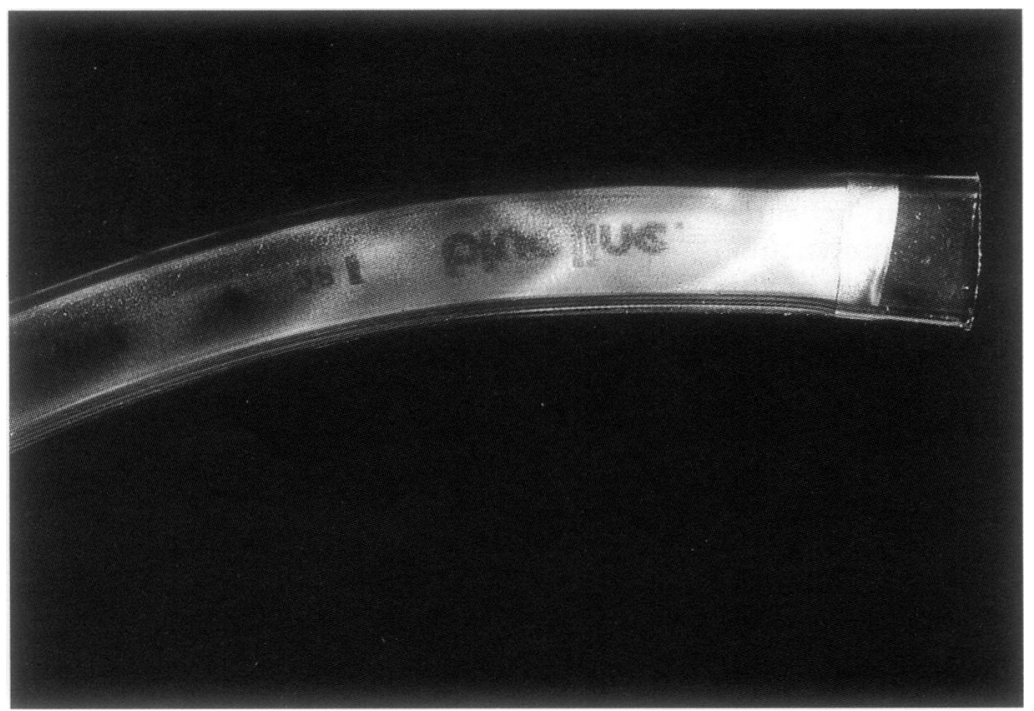

Figure 3 Pattern of powder deposition at the proximal end of the endotracheal tube after multiple actuations from the Turbohaler. mouthpiece (fig 1). The "spaceship" permits loading of the dosing channels without removing it from the device. Uncut $32 \mathrm{~cm}$ straight endotracheal tubes (Portex, Hythe, UK) with an internal diameter of $9 \mathrm{~mm}$ were used for all the experiments.

Initial experiments $(n=6)$ were performed in which an Astra multistage liquid impinger (Copley, Nottingham, UK) was used to assess both the quantity of drug and particle size distribution of the drug particles. The Turbohaler with the endotracheal tube attached was inserted into the inlet of the multistage liquid impinger and air was drawn through the system at a rate of $60 \mathrm{l} / \mathrm{min}$. When inflated, the endotracheal tube cuff provided a good seal when inserted into the inlet of the multistage liquid impinger, ensuring that the entire air flow $(60 \mathrm{l} / \mathrm{min})$ passed through the Turbohaler. The location of particle deposition is determined by the aerodynamic size of the particle. Those depositing on stages 3 and 4 represent particles of $<6.8 \mu \mathrm{m}$ in diameter and are often arbitrarily referred to as being in the "respirable range".

Ten actuations were used for each experiment to ensure detectable quantities of drug on each stage of the multistage liquid impinger. Each stage was washed with $50 \mathrm{ml}$ of ethanol and the amount of budesonide deposited was measured using spectrophotometer $(\lambda=243 \mathrm{~nm})$. The results were compared with results from a standard $200 \mu \mathrm{g}$ Turbohaler measured in the same way.

Further experiments $(n=15)$ were then performed with the Turbohaler placed in a ventilator circuit between the manifold and endotracheal tube as shown in fig 2. Drug delivered via the endotracheal tube was collected on a filter (Kendall Curity anaesthesia filters) placed between the end of the tube and a model lung. A Servo 900C (Siemens, Sweden) ventilator was used with the tidal volume set at $500 \mathrm{ml}$ and an inspiratory time of 0.5 seconds. A total of ten actuations were used for each experiment to ensure adequate quantities of drug on the filter. The pause button on the ventilator permits the Turbohaler to be loaded between inspiratory flows and potentially allows a pause for breath holding at the end of inspiration.

Each filter was washed with $100 \mathrm{ml}$ of ethanol and the budesonide was measured using a spectrophotometric method.

\section{Results}

The mean (SD) total dose from the standard Turbohaler including that retained on the mouthpiece was $204 \cdot 1(19 \cdot 6) \mu \mathrm{g}$. The dose collected by the multistage liquid impinger was $142.6(16.4) \mu \mathrm{g}$ and the dose of particles $<6.8 \mu \mathrm{m}$ in diameter was $91.2(8.9) \mu \mathrm{g}$. With the endotracheal tube attached, the mean (SD) quantity of drug delivered to the multistage liquid impinger was $60 \cdot 4(4 \cdot 8) \mu \mathrm{g}$ per actuation and the dose of particles $<6.8 \mu \mathrm{m}$ in diameter was $46.8(3.2) \mu \mathrm{g}$. The total dose of drug delivered to the filter at the distal end of the 
endotracheal tube when included in the ventilator circuit was $41 \cdot 1(3 \cdot 7) \mu \mathrm{g}$ per actuation.

Figure 3 illustrates the pattern of powder deposition at the proximal end of the endotracheal tube following multiple actuations, illustrating that the powder continues to spiral after leaving the mouthpiece.

\section{Discussion}

These results suggest that drug from dry powder delivery systems can be delivered efficiently via endotracheal tubes and that such systems may be valuable in the intensive care setting. Results with the multistage liquid impinger indicate that as much as $30 \%$ of the nominal dose can be delivered via an endotracheal tube. Although the total dose on stages 3 and 4 is reduced compared with the standard Turbohaler, these results also suggest that the tubing is particle selective in that the ratio of drug on stages $(3+4)$ to $(1+2)$ increases from $1 \cdot 77$ to 1 to 3.4 to 1 when delivered via the tube.

Air flow through the Turbohaler in the multistage liquid impinger experiments is essentially a square wave with flow reaching $601 / \mathrm{min}$ very rapidly which may not be the case in a ventilator circuit. Using an arbitrary tidal volume of $500 \mathrm{ml}$, delivery to the filter of $20 \%$ was still achieved in the ventilator filter study. Deposition studies assessing drug delivery from jet nebuliser and metered dose inhaler systems have found that the lung dose for intubated patients is generally in the range $1-6 \% .{ }^{147} \mathrm{It}$ has recently been shown in vitro that the use of tubing to effectively extend the actuator beyond the tip of the endotracheal tube can deliver doses approaching $100 \%$ to the lower respiratory tract. ${ }^{16}$ Unfortunately much of the aerosol is likely to impact directly on respiratory epithelium just beyond the endotracheal tube, and damage to the epithelium is likely to occur due to the impaction of high concentrations of propellants and surfactants such as oleic acid. ${ }^{17}$

Previous work has found that in vitro studies have overestimated lung doses from both metered dose inhaler ${ }^{610}$ and nebuliser ${ }^{4}$ based systems. It is likely that these experiments also overestimate the dose that would be delivered if this powder based system was used with intubated patients, particularly as there are a number of potential problems that should be considered. The gases in the ventilator circuit were not humidified in these experiments and the use of humidification might reduce drug delivery as it does for jet nebulisers. ${ }^{18}$ Furthermore, clean dry endotracheal tubes were used. From fig 3 it is evident that the powder continues to spiral along the inner surface of the tube for several centimetres and, if this surface was wet, increased drug loss is likely. Beyond this the powder is presumably reentrained into the air flow, so it is important to use a clean dry extension to connect the mouthpiece to the endotracheal tube and to use non-humidified gases during the administration of the aerosol.

The system we describe is easy to use and our initial results indicate that relatively efficient drug delivery is possible using a dry powder delivery sysem. We would suggest that this system is worthy of further assessment in the clinical setting and may prove to be a useful addition to drug delivery systems in the intensive care unit.

1 MacIntyre NR, Silver RM, Miller CW, Schuker F, Coleman E. Aerosol delivery in intubated, mechanically ventilated patients. Crit Care Med 1985;13:81-4.

2 Aherns RC, Ries RA, Popendorf W, Wiese JA. The delivery of therapeutic aerosols through endotracheal tubes. Pediatr Pulmonol 1986;2:19-26.

3 Manthous CA, Hall JB. Administration of therapeutic aerosols to mechanically ventilated patients. Chest 1994;106 560-71.

4 Harvey CJ, O'Doherty MJ, Page CJ, Thomas SHL, Nunan TO, Treacher DF. Effect of a spacer on pulmonary aerosol deposition from a jet nebuliser during mechanical ventilation. Thorax 1995;50:50-3.

5 Hess D. Aerosol bronchodilator delivery during mechanical ventilation. Nebulizer or inhaler? Chest $1991 ; 100: 1103-4$

6 Newhouse MT, Fuller HD. Rose is a rose is a rose? Aerosol therapy in ventilated patients: nebulizers versus metered therapy in ventilated patients: nebulizers versus metered Dis 1993;148:1444-6.

7 Fuller HD, Dolovich MB, Posmituck G, Wong Pack W, Newhouse MT. Pressurised aerosol versus jet aerosol to
Nes mechanically ventilated patients. Am Rev Respir Dis 1990; 141:440-4.

8 Gay PC, Patel HG, Nelson SB, Gillies B, Hubmayr RD. Metered dose inhalers for bronchodilator delivery in intubated mechanically ventilated patients. Chest 1991;99: 66-71.

9 Rau JL, Harwood RJ, Groff JL. Evaluation of a reservoir device for metered-dose bronchodilator delivery to intubated adults. An in vitro study. Chest 1992;102:924-30.

10 O'Doherty MJ, Thomas SHL, Page CJ, Treacher DF Nunan TO. Delivery of a nebulized aerosol to a lung model during mechanical ventilation. Am Rev Respir Dis model during mech

11 Dahlback M, Wollmer P, Drefeldt B, Jonson B. Controlled aerosol delivery during mechanical ventilation. $f$ Aerosol Med 1989;4:339-47.

12 Thomas SHL, O'Doherty MJ, Page CJ, Treacher DF, Nunan TO. Delivery of ultrasonic nebulized aerosols to a lung model during mechanical ventilation. Am Rev Respir Dis 1993;148:872-7.

13 Manthous CA, Hall JB, Schmidt GA, Wood LDH. Metered dose inhaler versus nebulized albuterol in mechanically ventilated patients. Am Rev Respir Dis 1993;148:1567-71.

14 Beasley R, Rafferty A, Holgate ST. Adverse reactions to the non-drug constituents of nebuliser solution. $\mathrm{Br} \mathcal{f}$ Clin Pharmacol 1988;25:283-7.

15 Engel T. Patient-related side effects of CFC propellants. $\mathcal{f}$ Aerosol Med 1991;4:163-7.

16 Taylor RH, Lerman J, Chambers C, Dolovich M. Dosing efficiency and particle-size characteristics of pressurised metered dose inhaler aerosols in narrow catheters. Chest 1993;103:920-4.

17 Spah-Schopfer IA, Lerman J, Cutz E, Mewhouse MT, Dolovich M. Proximate delivery of a large experimental dose from salbutamol MDI induces epithelial airway lesions in intubated rabbits. Am Rev Respir Crit Care Med 1994;150:790-4.

18 O'Riordan TG, Greco MJ, Perry RJ, Smalldone GC. Nebulizer function during mechanical ventilation. $A m$ Rev Respir Dis 1992;145:1117-22. 